\title{
GERMINACIÓN Y CRECIMIENTO INICIAL DE SEMILLAS DE MAÍZ CON ENVEJECIMIENTO NATURAL ${ }^{1}$
}

\author{
Germán Fernando Gutiérrez-Hernández $z^{2}$ Juan Virgen-Vargas ${ }^{3}$, José Luis Arellano-Vázquez ${ }^{3}$
}

\begin{abstract}
RESUMEN
Germinación y crecimiento inicial de semillas de maíz con envejecimiento natural. Para detectar y cuantificar los daños causados por el envejecimiento natural sobre algunos eventos iniciales de la germinación seminal y del crecimiento inicial de las plántulas, se realizó en el Laboratorio de Biotecnología de la Unidad Profesional Interdisciplinaria de Biotecnología (UPIBI) del Instituto Politécnico Nacional, Ticomán, México, durante el 2005, el análisis de componentes físicos y fisiológicos de semillas de dos edades (19 y seis años), de dos cruzas simples de maíz y de sus líneas progenitoras. Las semillas estuvieron almacenadas sin control de temperatura ni humedad. Se usó un diseño de bloques al azar con dos repeticiones y 10 semillas como unidad experimental. En cada unidad se evaluó el contenido de humedad, integridad del pericarpio, lixiviación de solutos, viabilidad y protrusión radicular. Los componentes físicos (contenido de humedad e integridad del pericarpio) permanecieron sin variaciones significativas durante los años de almacenamiento de las semillas; sin embargo, las fisiológicas (protrusión radicular y viabilidad) se afectaron $(\mathrm{p} \leq 0,05)$ hasta hacerse nulas en semillas de mayor edad, en las cuales también el lixiviado de solutos fue más profuso $(\mathrm{p} \leq 0,05)$. Se detectaron correlaciones significativas para viabilidad con protrusión radicular $(0,98 * *)$ y también con lixiviación de solutos $\left(-0,66^{*}\right)$, así como entre lixiviación de solutos y protrusión radicular $(-0,66 *)$.
\end{abstract}

Palabras clave: Longevidad, senescencia, vigor seminal, deterioro natural, fisiología de semillas.

\begin{abstract}
Germination and initial growth of naturally-aged maize seeds. In order to detect and to quantify the damage caused by natural ageing of seeds on events involved in the initial stages of maize germination, it was made at Laboratory of Biotechnology, Instituto Politécnico Nacional, Ticomán, México, in 2005, a physical and physiological analysis of seeds of two ages, 6 and 19 years old, of two single crosses of the maize hybrids $\mathrm{H} 28$ and $\mathrm{H} 30$, and their inbred parental lines, were performed. The seeds were stored without control of temperature and humidity. Our study had a total of 12 treatments. A randomly blocked design with two replications of 10 seeds each was used. Variables evaluated were: content of humidity, pericap integrity, solute lixiviation, seed viability and radicle protrusion. The results indicated that the physical variables (humidity content and pericarp integrity) remained unchanged during the seed storage period; however, the physiological variables (radicle protrusion and viability) were affected $(\mathrm{p} \leq 0.05)$ by ageing. Solute lixiviation was significantly $(\mathrm{p} \leq 0.05)$ profuse in 19 year old seeds. Correlations between viability and radicle protrusion $(0.98 * *)$, viability and leaching out solutes ($0.66 *)$, as well as between leaching out solutes and radical protrusion $(-0.66 *)$, were significant.
\end{abstract}

Key words: Longevity, senescence, vigor, natural ageing, seed physiology.

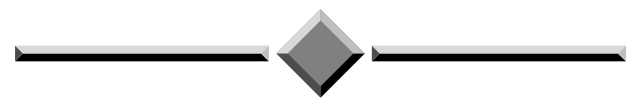

1 Recibido: 14 de febrero, 2007. Aceptado: 29 de agosto, 2007. Proyecto de investigación interinstitucional. Instituto Politécnico Nacional - Instituto Nacional de Investigaciones Forestales, Agrícolas y Pecuarias (IPN - INIFAP).

2 Bioprocesos, Unidad Profesional Interdisciplinaria de Biotecnología (UPIBI), Instituto Politécnico Nacional (IPN). Av. Acueducto s/n. 07340, La Laguna Ticomán. México, D. F. Tel. (01-55) 5729-6000, ext. 56343. Correo electrónico: ggutierrez@acei.upibi.ipn.mx (Autor para correspondencia).

3 Programas de Semillas y Maíz, respectivamente, Campo Experimental Valle de México (CEVAMEX), Instituto Nacional de Investigaciones Forestales, Agrícolas y Pecuarias (INIFAP). Apartado Postal 10.56230, Chapingo, México. Correos electrónicos: jvirgen_vargas@hotmail. com y arevajolu@yahoo.com.mx, respectivamente. 


\section{INTRODUCCIÓN}

Se ha demostrado la disminución paulatina del potencial fisiológico de las semillas de maíz (Zea mays L.), ocasionada por el envejecimiento natural, y misma que merma progresivamente la capacidad germinativa, la velocidad de crecimiento inicial de la plántula y la tolerancia a condiciones adversas (Marcos-Filho y McDonald 1998; McDonald 1999). Tales efectos están asociados con alteraciones bioquímicas evidenciadas durante las primeras horas de imbibición de los tejidos seminales (Cruz et al. 1995), y se expresan primero como reducciones en el desempeño germinativo y en el vigor de las semillas y, posteriormente, al nivel del ADN (Marcos-Filho y McDonald 1998).

El vigor es una característica genética de la planta expresada a nivel de semilla, que es afectada por factores exógenos como la nutrición de la planta madre, daños mecánicos ocasionados durante la cosecha, el procesamiento y el almacenamiento (McDonald 1998).

Los síntomas de la semilla deteriorada incluyen: pérdida de compuestos solubles por la anómala permeabilidad de las membranas celulares, reducción de la actividad enzimática y producción de sustancias tóxicas como ácidos grasos libres, todo lo cual repercute en una reactivación deficiente del crecimiento embrional (Anderson 1973). La escasa o nula funcionalidad de las membranas seminales es consecuencia de la peroxidación de los lípidos que las constituyen, lo que se traduce en su desorganización y pérdida de actividad metabólica (Wilson y McDonald 1986).

Además, la excesiva permeabilidad provoca la pérdida masiva de azúcares, iones inorgánicos y aminoácidos, entre otros componentes celulares, y algunos de ellos podrían quedar en concentraciones inferiores a las óptimas para respiración, actividad enzimática y síntesis de macromoléculas (Bewley y Black 1994). El envejecimiento o deterioro de las semillas es un fenómeno complejo, que difiere entre genotipos, es influenciado por factores ambientales y biológicos, y no ocurre uniformemente en las semillas, aún dentro de un mismo lote (McDonald 1999).

El objetivo del presente estudio fue identificar y cuantificar la repercusión del envejecimiento natural sobre los procesos físicos (contenido de humedad y daño mecánico) y fisiológicos (viabilidad, protrusión radicular y lixiviación de solutos) implicados en la germinación de las semillas de dos cruzas simples de maíz, así como en las de sus respectivos progenitores.

\section{MATERIALES Y MÉTODOS}

Se emplearon semillas de los híbridos de maíz: H28 y 30, así como las de sus respectivos progenitores, pertenecientes al Programa de Semillas del Campo Experimental Valle de México (CEVAMEX), del Instituto Nacional de Investigaciones Forestales, Agrícolas y Pecuarias (INIFAP).

Las semillas estuvieron almacenadas sin control de temperatura ni humedad. El clima del lugar corresponde a un templado húmedo, con lluvias en verano, el cual es fresco y de escasa oscilación térmica $\left[\mathrm{C}\left(\mathrm{w}_{0}\right)\right.$ (W) b (i') g], con una precipitación media de $670 \mathrm{~mm}$ anuales y $15,2{ }^{\circ} \mathrm{C}$ de temperatura media anual, y con una altitud de $2.249 \mathrm{msnm}$ (García 1973).

De cada híbrido (de la cruza simple y de sus progenitores) se eligieron semillas con 19 y seis años de edad, para integrar un total de 12 tratamientos (Cuadro 1).

El estudio se realizó en el Laboratorio de Biotecnología de la Unidad Profesional Interdisciplinaria de Biotecnología (UPIBI) del Instituto Politécnico Nacional (IPN), durante el 2005.

\section{Evaluaciones físicas}

\section{Contenido de humedad}

Para obtener el contenido de humedad, se registraron los pesos fresco y seco de las semillas; para este último se secaron previamente a $70{ }^{\circ} \mathrm{C}$ por $72 \mathrm{~h}$. El contenido de humedad se calculó de acuerdo con la fórmula (López 1995):

$$
H(\%)=\frac{P F-P S}{P F} \times 100
$$


Donde: $\mathrm{H}=$ Contenido de humedad de la semilla $(\%), \mathrm{PF}=$ Peso fresco de la semilla $(\mathrm{g}), \mathrm{y}$ PS = Peso seco de la semilla $(\mathrm{g})$.

Cuadro 1. Cruzas simples de maíz ("H28” y "H30"), así como de sus líneas progenitoras, de dos edades y almacenadas bajo ambiente natural. CEVAMEXINIFAP, Chapingo, México, 2005.

\begin{tabular}{clcl}
\hline $\begin{array}{c}\text { Trata- } \\
\text { miento }\end{array}$ & \multicolumn{1}{c}{ Línea } & $\begin{array}{c}\text { Edad } \\
\text { (años) }\end{array}$ & \multicolumn{1}{c}{ Genealogía } \\
\hline \multicolumn{4}{c}{ H28 } \\
\hline 1 & M17 & 19 & Mich21 Comp. 1-7-2 \\
2 & M18 & 19 & Mich21 Comp. 1-27-2 \\
3 & M17 X M18 & 19 & Cruza simple \\
4 & M17 & 6 & Mich21 Comp. 1-7-2 \\
5 & M18 & 6 & Mich21 Comp. 1-27-2 \\
6 & M17 X M18 & 6 & Cruza simple \\
\hline & & H30 & \\
\hline 7 & M27 & 19 & Mich21-181-14-1 \\
8 & M28 & 19 & Mich21-183 \\
9 & M27X M28 & 19 & Cruza simple \\
10 & M27 & 6 & Mich21-181-14-1 \\
11 & M28 & 6 & Mich21-183 \\
12 & M27X M28 & 6 & Cruza simple \\
\hline
\end{tabular}

\section{Daño mecánico}

Con la prueba del verde rápido (López 1995) se determinaron los daños mecánicos que las semillas presentaron en el pericarpio. Para ésto, las semillas se sumergieron en $10 \mathrm{ml}$ de la solución del colorante verde rápido $(2 \%, \mathrm{p} / \mathrm{v})$, a temperatura ambiente por 30 min; luego se lavaron con agua destilada y se secaron superficialmente sobre toallas de papel. Cada semilla se inspeccionó mediante un microscopio estereoscópico y el daño en el pericarpio se manifestó como porciones teñidas intensamente de un verde oscuro.

\section{Evaluaciones fisiológicas}

\section{Viabilidad}

La viabilidad se determinó mediante la prueba de tetrazolio (Delouche et al. 1971). Las semillas fueron embebidas en agua destilada por $12 \mathrm{~h}$ a temperatura ambiente y luego se disecaron longitudinalmente por el eje embrional; se eligieron las mitades de las semillas que mostraron mayor definición de sus componentes morfológicos, y se mantuvieron sumergidas en agua destilada, para posteriormente colocarlas en cajas petri con el corte hacia abajo y agregarles $10 \mathrm{ml}$ de la solución de cloruro-2-3, 5-trifenil tetrazolio $(1 \%, \mathrm{p} / \mathrm{v})$.

Las cajas petri permanecieron en incubación por 4 h a $25 \pm 2{ }^{\circ} \mathrm{C}$, y en seguida, se reemplazó la solución de tetrazolio con agua destilada. La lectura de viabilidad se hizo en las estructuras embrionales, con un microscopio estereoscópico. A cada semilla se le asignó uno de los 16 casos de la clave de interpretación para la prueba de tetrazolio en maíz (Delouche et al. 1971).

\section{Protrusión radicular}

La aptitud para protruir se evaluó cada 24 h, a partir del inicio de la hidratación de las semillas y hasta ocho días después. Para esta prueba las semillas se incubaron a $25{ }^{\circ} \mathrm{C}$, colocadas sobre papel filtro en cajas petri al que se adicionaron $10 \mathrm{ml}$ de agua destilada, volumen que se procuró mantener constante. Se consideró que la radícula había protruido de la semilla cuando alcanzaba una longitud aproximada de 3 mm (López 1995).

\section{Lixiviación de solutos}

Las semillas se limpiaron escrupulosamente en su superficie y se colocaron en tubos de ensayo (13 x 150 $\mathrm{mm}$ ) que contenían $15 \mathrm{ml}$ de agua destilada. La lixiviación se determinó a $0,2,4,6,8$ y 24 h posteriores a la imbibición, mediante la absorbancia del líquido de imbibición en un espectrofotómetro (Beckman DU-350, California, USA) a una longitud de onda de $580 \mathrm{~nm}$, la cual se determinó en experimentos previos al presente.

\section{Análisis estadístico}

Los datos se analizaron bajo un diseño completamente al azar, con 12 tratamientos (Cuadro 1), dos repeticiones por tratamiento y con 10 semillas como unidad experimental. La comparación de medias se hizo mediante la prueba de Tukey ( $\mathrm{p} \leq 0,05)$. Los datos porcentuales se normalizaron mediante la transfor- 
mación por arcoseno antes de realizar los análisis de varianza y la comparación de medias correspondientes (Reyes 1980). Además, se calcularon los coeficientes de correlación lineal entre las variables.

\section{RESULTADOS Y DISCUSIÓN}

Se detectaron diferencias significativas en el análisis de varianza para todas las variables, excepto para daño mecánico. El contenido de humedad de las semillas fue similar entre genotipos y entre edades, con valores entre 8,6 y $9,5 \%$, excepto en el tratamiento 1 (línea M17 con 19 años de almacenamiento) que tuvo menor cantidad de agua (4,2 \%). Esta diferencia se atribuye a alguna situación particular del almacenamiento, puesto que no pudo ser genotípica, porque la misma línea con seis años de edad (tratamiento 4) presentó un porcentaje de humedad similar a los demás tratamientos. Es decir, el proceso de envejecimiento no repercutió en esta variable.

Tampoco en la integridad (daño) del pericarpio hubo efectos estadísticos por la senescencia de las semillas; no obstante, se advierte la tendencia en ambas cruzas simples y edades (tratamientos 3, 6, 9 y 12), a denotar menor proporción de daños mecánicos, independientemente de su edad (Figura 1), que la línea hembra de cada cruza (tratamientos $1,4,7$ y 10).

El que se haya mantenido constante el contenido de humedad de las semillas y la ausencia de daño mecánico significativo en el pericarpio, a través del prolongado almacenamiento (19 y seis años) sin control de temperatura ni humedad, evidencia que el manejo postcosecha y almacenamiento fueron óptimos en cuanto a estas variables físicas. Sin embargo, ésto no fue suficiente para preservar su aptitud fisiológica, puesto que ocurrió una declinación del potencial germinativo de las semillas a través del añejamiento, hasta llegar a ser nula la viabilidad y la protrusión radicular en semillas de 19 años de edad (tratamientos 1, 2, 3, 7, 8 y 9) (Cuadro 2).

La lixiviación de solutos de las semillas se estabilizó a partir de las 6 h de imbibición en todos los tratamientos (Figura 2), pero con diferencias significativas entre genotipos (Cuadro 2). Por ello se eligió este lapso de hidratación de las semillas para efectuar la comparación estadística de la pérdida de solutos celulares (Cuadro 2), la cual se estimó mediante los valores de absorbancia en los lixiviados presentes en el agua de
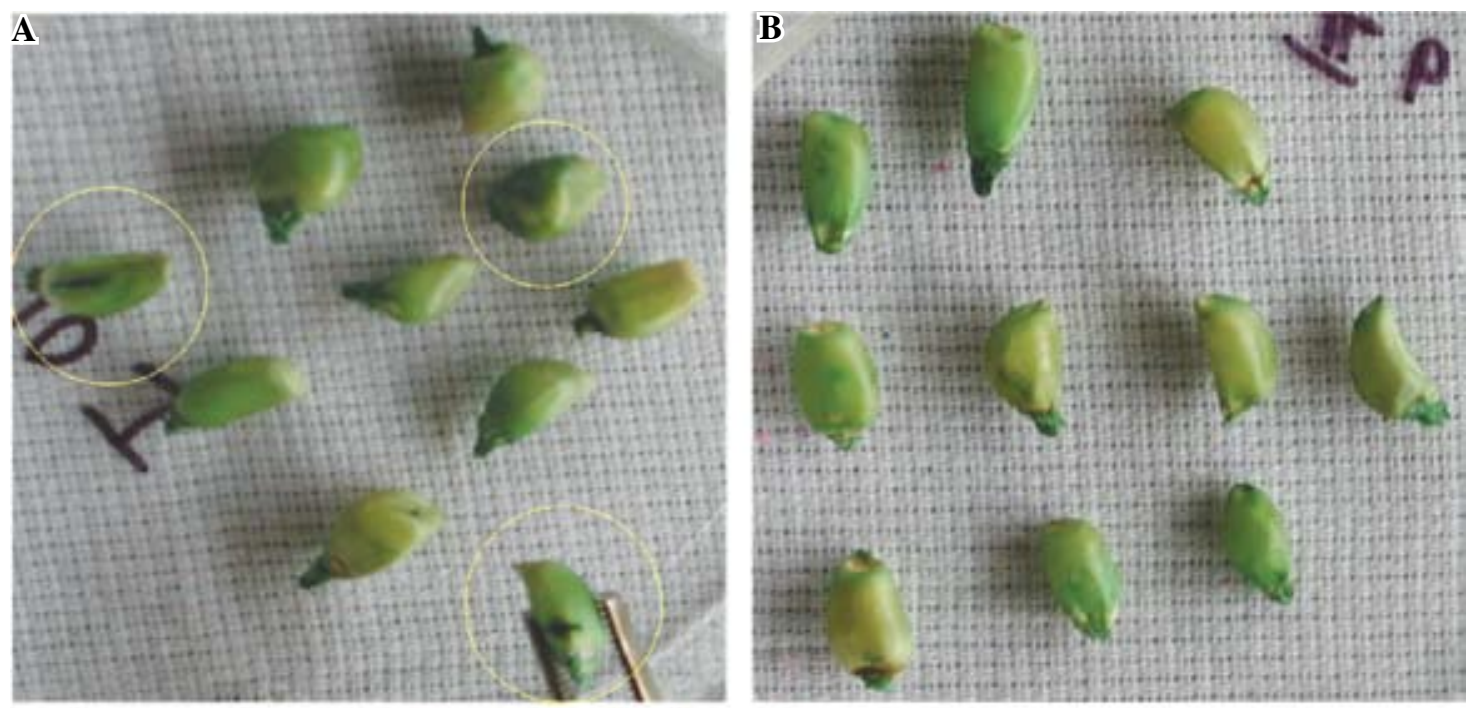

Figura 1. Tinción con solución Verde Rápido $(2 \%, \mathrm{p} / \mathrm{v})$ para determinar daños en el pericarpio de la semilla maíz. La tonalidad verde oscuro en las zonas dentro de círculos evidencia daño mecánico en el pericarpio. A) M27 con seis años de edad, tratamiento 10), mientras que las semillas sin daños exhiben una coloración uniforme. B) M17 x M18 con 19 años de edad, tratamiento 3). Unidad Profesional Interdisciplinaria de Biotecnología del Instituto Politécnico Nacional (UPIBI-IPN), México. 2005. 
Cuadro 2. Comparación de medias de las variables físicas y fisiológicas de semillas de maíz. Unidad Profesional Interdisciplinaria de Biotecnología del Instituto Politécnico Nacional (UPIBI-IPN), México. 2005.

\begin{tabular}{|c|c|c|c|c|c|}
\hline Tratamiento & $\begin{array}{l}\text { Contenido de } \\
\text { humedad }(\%)\end{array}$ & $\begin{array}{c}\text { Daño mecánico } \\
(\%)\end{array}$ & Viabilidad (\%) & $\begin{array}{c}\text { Protrusión } \\
\text { radicular }(\%)\end{array}$ & $\begin{array}{c}\text { Lixiviación de solutos } \\
\left(A \times \mathbf{1 0}^{-4}\right)^{\dagger}\end{array}$ \\
\hline 1 & $4,2 \quad b$ & $37 \mathrm{a}$ & $\begin{array}{ll}0 & \mathrm{~b}\end{array}$ & $0 \quad b$ & $5.572 \mathrm{ab}$ \\
\hline 2 & 8,9 a & $30 \mathrm{a}$ & $0 \quad \mathrm{~b}$ & $0 \quad \mathrm{~b}$ & $4.790 \mathrm{ab}$ \\
\hline 3 & 9,5 a & $13 \mathrm{a}$ & $0 \quad b$ & $0 \quad b$ & $2.125 \mathrm{ab}$ \\
\hline 4 & 9,5 a & $40 \mathrm{a}$ & $85 \mathrm{a}$ & $81 \mathrm{a}$ & $1.318 \mathrm{ab}$ \\
\hline 5 & 9,4 a & 9 a & 90 a & 75 a & $843 \mathrm{~b}$ \\
\hline 6 & $9,0 \quad \mathrm{a}$ & $12 \mathrm{a}$ & $92 \mathrm{a}$ & 98 a & $919 \mathrm{~b}$ \\
\hline 7 & 9,2 a & 19 a & $0 \quad \mathrm{~b}$ & $0 \quad b$ & $4.949 \mathrm{ab}$ \\
\hline 8 & $9,0 \quad \mathrm{a}$ & $1 \mathrm{a}$ & $\begin{array}{ll}0 & \mathrm{~b}\end{array}$ & $\begin{array}{ll}0 & b\end{array}$ & $5.881 \mathrm{ab}$ \\
\hline 9 & 8,6 a & $7 \mathrm{a}$ & $3 \mathrm{~b}$ & $\begin{array}{ll}0 & \mathrm{~b}\end{array}$ & $6.154 \mathrm{a}$ \\
\hline 10 & 8,7 a & $44 \mathrm{a}$ & $81 \mathrm{a}$ & $77 \mathrm{a}$ & $5.572 \mathrm{ab}$ \\
\hline 11 & 9,5 a & $19 \mathrm{a}$ & $70 \mathrm{a}$ & $90 \mathrm{a}$ & $2.544 \mathrm{ab}$ \\
\hline 12 & $8,7 \quad$ a & $22 \mathrm{a}$ & $75 \mathrm{a}$ & $90 \mathrm{a}$ & $3.324 \mathrm{ab}$ \\
\hline
\end{tabular}

${ }^{\dagger}$ Absorbancia del lixiviado a las 6 h de imbibición.

Medias con la misma letra, en cada columna, son estadísticamente iguales (Tukey, 0,05).

imbibición de las semillas. Las semillas de mayor edad (19 años) lixiviaron significativamente mayor cantidad de metabolitos que las más jóvenes (seis años); la ma- yor lixiviación ocurrió en la cruza simple M27 x M28 con 19 años, y la menor en la línea M18, con seis años de edad (tratamientos 9 y 5 , respectivamente).

A

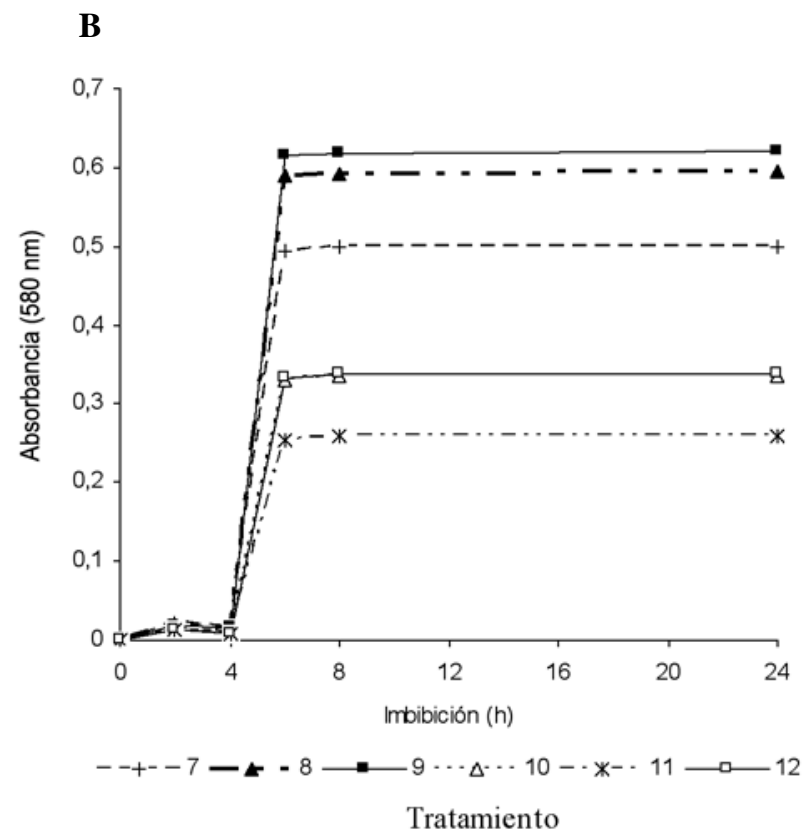

Figura 2. Cinética de lixiviación de solutos de las semillas de los híbridos de maíz "H28” (A) y "H30” (B), y de sus respectivos progenitores. Unidad Profesional Interdisciplinaria de Biotecnología del Instituto Politécnico Nacional (UPIBI-IPN), México. 2005. 
La salida de metabolitos celulares tendió a ser menor o intermedia en las cruzas simples en comparación con sus líneas progenitoras, pero sin alcanzar diferencias significativas. El comportamiento de estos genotipos en lixiviación de solutos coincide con el que exhibieron para daño mecánico, y podrían ser manifestaciones heteróticas de escasa magnitud, debido a que las cruzas simples poseen una base genética similar, así como un reducido nivel de endogamia en sus líneas progenitoras (Cuadro 1), circunstancia que habría que corroborar con mayor tamaño de muestra y más repeticiones.

Los procesos fisiológicos de viabilidad y protrusión radicular (Cuadro 2) fueron nulos en las semillas de mayor edad, comportamiento acorde con lo reportado por varios autores (Cruz et al. 1995; Pérez y Martínez 1994), quienes aducen que el avance de la edad induce alteraciones progresivas e irremisibles en diferentes niveles metabólicos (bioquímico, fisiológico y molecular), las cuales menoscaban la capacidad fisiológica de las semillas para germinar e incluso eliminan todo signo de actividad metabólica (viabilidad).

En la prueba de tinción con tetrazolio se detectaron zonas sin actividad metabólica distribuidas al azar en las estructuras del embrión, aunque en las semillas de 19 años de edad, fue predominantemente en el meristemo radicular (Figura 3), donde no se produjo el formazán, compuesto rojo insoluble que se produce al
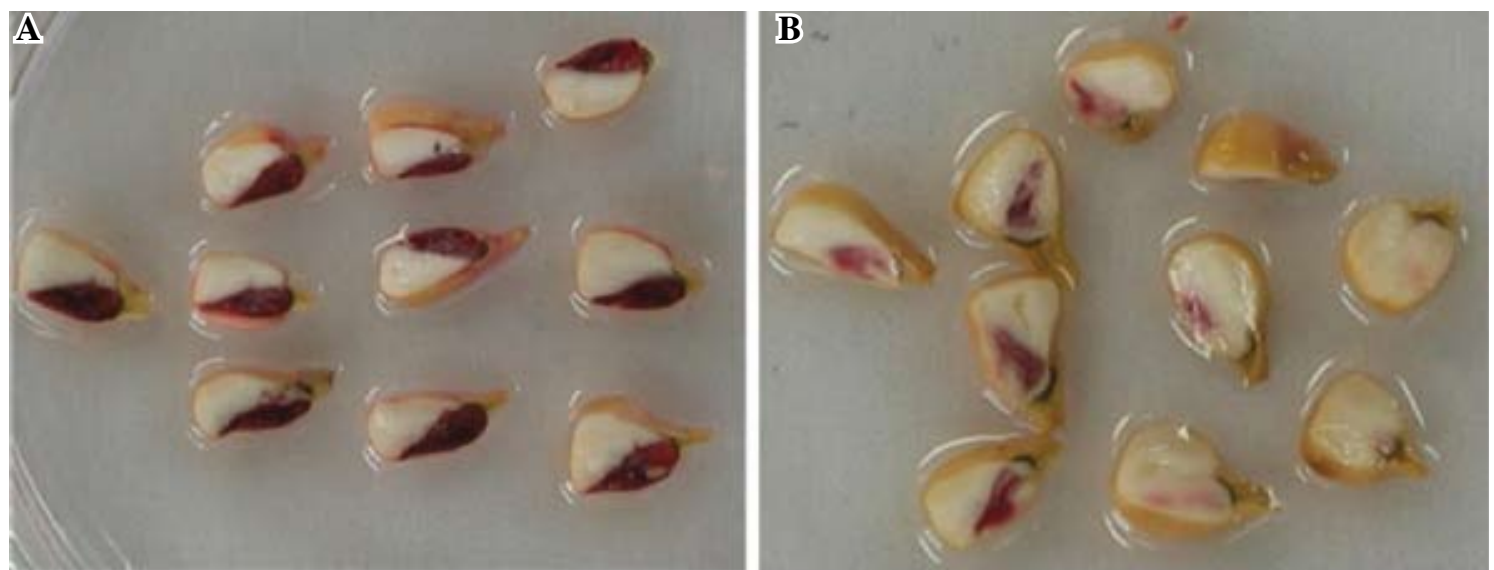

Figura 3. Prueba de viabilidad con tetrazolio $(1 \%, \mathrm{p} / \mathrm{v})$. El color rojo intenso del embrión denota alta viabilidad de la semilla (A, M17 con seis años de edad, tratamiento 4), mientras que la ausencia de color indica escasa o nula actividad metabólica, particularmente ésto se observa en la zona de la radícula (B, M17 con 19 años de edad, tratamiento 1). Unidad Profesional Interdisciplinaria de Biotecnología del Instituto Politécnico Nacional (UPIBI-IPN), México. 2005.

Cuadro 3. Coeficientes de correlación lineal entre las variables físicas y fisiológicas del estudio. Unidad Profesional Interdisciplinaria de Biotecnología del Instituto Politécnico Nacional (UPIBI-IPN), México. 2005.

\begin{tabular}{lcccc}
\hline & $\begin{array}{c}\text { Contenido de } \\
\text { humedad }\end{array}$ & $\begin{array}{c}\text { Daño } \\
\text { mecánico }\end{array}$ & Viabilidad & $\begin{array}{c}\text { Protrusión } \\
\text { radicular }\end{array}$ \\
\hline Daño mecánico & $-0,33 \mathrm{~ns}$ & & & \\
Viabilidad & $0,33 \mathrm{~ns}$ & $0,23 \mathrm{~ns}$ & & \\
Protrusión radicular & $0,32 \mathrm{~ns}$ & $0,24 \mathrm{~ns}$ & $0,98 * *$ & $-0,66 *$ \\
$\begin{array}{l}\text { Lixiviación de } \\
\text { solutos }\end{array}$ & $-0,41 \mathrm{~ns}$ & $0,02 \mathrm{~ns}$ & $-0,66 *$ & \\
\hline
\end{tabular}

$*, * *=$ Significativo $(\mathrm{P} \leq 0,05$ y 0,01 , respectivamente $)$; ns = No significativo. 
reducirse la solución de tetrazolio con los electrones del proceso respiratorio de las células activas al inicio de su hidratación (Moore y Goodsell 1965). La carencia de actividad respiratoria en la región radicular ha sido ya documentada en semillas envejecidas de manera natural (Cruz et al. 2003) y ésto explicaría el alto grado de asociación $(0,98 * *)$ entre viabilidad y protrusión radicular en el análisis de correlación (Cuadro 3).

Tal comportamiento en la lixiviación de solutos de las semillas, sugiere a la disminución o pérdida de funcionalidad de la membrana celular, como un signo de senescencia de semillas, mecanismo ya reportado por Wilson y McDonald (1986). Esta posibilidad se respalda con los valores de correlación (Cuadro 3), en los cuales la lixiviación de solutos celulares se asoció inversa y significativamente con viabilidad $(-0,66 *)$ y con protrusión radicular $(-0,66 *)$.

Fisiológicamente estas manifestaciones podrían interpretarse como una secuencia de eventos de deterioro que comienza con problemas de funcionalidad en las membranas seminales, lo que ocasiona un flujo excesivo de constituyentes celulares, evidenciado éste por los altos valores de absorbancia y la consecuente pérdida de metabolitos, cuya magnitud puede restringir al proceso germinativo (Bewley y Black 1994).

\section{CONCLUSIONES}

El envejecimiento de las semillas no repercutió en su contenido de humedad ni sobre la integridad del pericarpio, pero sí menoscabó su potencial metabólico, en términos de viabilidad y de protrusión radicular.

Los valores de correlación evidenciaron que el potencial metabólico de las semillas (viabilidad) repercutió directamente sobre su germinación; en tanto que la pérdida de metabolitos celulares (lixiviación), incidió de manera adversa en su germinabilidad.

La lixiviación de solutos de las semillas de mayor edad fue significativamente más cuantiosa, con respecto a las más jóvenes, por lo que es posible considerarla como signo de deterioro.

\section{AGRADECIMIENTOS}

A la Secretaría de Investigación y Posgrado del IPN (SIP:20060409) y a la Comisión de Operación y Fomento de Actividades Académicas del IPN, por el financiamiento; al INIFAP por dar acceso a su valioso germoplasma, y al Laboratorio de Biotecnología de la UPIBI por las facilidades otorgadas.

\section{LITERATURA CITADA}

ANDERSON, J. D. 1973. Metabolic changes associated with senescence. Seed Science and Technology 1:401-406.

BEWLEY, J. D.; BLACK, A. M. 1994. Seeds: Physiology of development and germination. 2 ed. Plenum Press. N. Y. USA. 445 p.

CRUZ, G. F.; GONZÁLEZ-H, V. A.; MOLINA-M., J.; VÁZQUEZ-R., J. M. 1995. Seed deterioration and respiration as related to DNA metabolism in germinating maize. Seed Science and Technology 23:477-486.

CRUZ-P., A. B.; GONZÁLEZ-H., V. A.; MENDOZA-C., M.; ORTEGA-D., M. L. 2003. Marcadores fisiológicos de la tolerancia al envejecimiento de semilla en maíz. Agrociencia 37:371-381.

DELOUCHE, J. C.; WAYNE S., T.; RASPET, M.; LIENHARD, M. 1971. Prueba de viabilidad de semillas con tetrazol. CRAT, AID. Buenos Aires, Argentina. 256 p.

LÓPEZ, H. A. 1995. Manual de prácticas de laboratorio de producción y tecnología de semillas. Fitotecnia, UACH. Chapingo, México. 29 p.

MARCOS-FILHO, J.; MCDONALD, M. B. 1998. Sensitivity of RAPD analysis, germination and vigour test to detect the intensity of deterioration of naturally and artificially aged soybean seeds. Seed Science and Technology 26:141-157.

MCDONALD, M. B. 1998. Seed quality assessment. Seed Science and Technology 8:265-275.

MCDONALD, M. B. 1999. Seed deterioration: physiology, repair, and assessment. Seed Science and Technology 27:177-237. 
MOORE, R. P.; GOODSELL, S. F. 1965. Tetrazolium test for predicting cold test performance of seed corn. Agron. J. 2:489-491.

PÉREZ, F. G.; MARTÍNEZ J. B. 1994. Introducción a la fisiología vegetal. Mundi-Prensa. México. 250 p.
REYES, C. P. 1980. Bioestadística aplicada: agronomía, biología, química. Ed. Trillas, México, D. F. 213 p.

WILSON, D. O.; MCDONALD, M. B. 1986. The lipid peroxidation model of seed ageing. Seed Science and Technology 27:177-237. 